\title{
Análise de Custo da Nuvem Computacional para a Execução de Algoritmos no Processamento Sísmico
}

\author{
Nicholas T. Okita ${ }^{1}$, Tiago A. Coimbra ${ }^{1}$, Edson Borin ${ }^{1}$ \\ ${ }^{1}$ Centro de Estudos de Petróleo (CEPETRO) \\ Universidade Estadual de Campinas (UNICAMP) \\ Caixa Postal 6052 - 13.083-970 - Campinas - SP - Brasil \\ nicholas.okita@ggaunicamp.com, tiago@ggaunicamp.com, edson@ic.unicamp.br
}

\begin{abstract}
Resumo. $O$ objetivo deste trabalho é a investigação do uso da nuvem computacional para a execução de algoritmos no processamento sísmico, visando minimizar o custo financeiro com o uso do serviço em nuvem contra a compra de máquinas físicas. Os resultados obtidos indicam que (i) as máquinas virtuais equipadas com GPU apresentam o menor custo/benefício para a aplicação executada, (ii) existe escalabilidade no desempenho e esta pode ser explorada para obter tempos de execuções menores e, por fim, (iii) o uso da nuvem permite que não seja necessária a compra de novas máquinas físicas.
\end{abstract}

\section{Introdução}

Nos dias atuais, tarefas que necessitam de alto poder computacional ganharam destaque devido ao processamento de dados cada vez maiores. Assim, o processamento de grandes volumes de dados em um tempo viável é um dos principais desafios na sísmica de exploração. Para tratar de tal desafio, a nível de software, algoritmos e métodos heurísticos mais eficientes foram criados para reduzir o tempo de execução. Entretanto, em nível de hardware, o processamento paralelo e em nuvem foram aprimorados a fim de reduzir o tempo de processamento.

Este trabalho visa o processamento em nuvem como uma alternativa para a compra de um novo hardware, o qual apresenta problemas como a incerteza de qual será o investimento inicial para que o mesmo não seja obsoleto até o fim do projeto, além da manutenção, instalação e uso dependendo do tamanho do desse hardware, por exemplo um cluster. Enquanto em nuvem, usando-se do modelo de negócio "Pay as you go", não existe essa preocupação. Pois, paga-se apenas a quantidade de tempo utilizado nas especificações de hardware desejadas.

Neste trabalho dois tópicos foram investigados, os quais são: (i) a busca do menor custo/benefício na escolha de máquinas em nuvem para o processamento sísmico; e (ii) a análise do investimento em hardware contra o uso do mesmo investimento em máquinas em nuvem.

O texto está organizado da seguinte forma: a seção 2 apresenta os recursos computacionais da nuvem utilizados, a descrição dos testes executados e os métodos de comparação. A seção 3 apresenta e discute os resultados dos experimentos realizados, obtendo informações sobre a otimização de custo de uso da nuvem computacional. Por fim, a seção conclusão apresenta as conclusões derivadas dos experimentos. 


\section{Materiais e métodos}

A nuvem computacional Azure, da Microsoft, foi utilizada para realização dos experimentos onde vários conjuntos de máquinas foram criados para comparar o desempenho e o custo do algoritmo do método common-midpoint (CMP) [Yilmaz 2001]. O método CMP foi implementado utilizando o modelo Spits da plataforma PYPITS [Borin et al. 2016]. O Spits é um modelo de programação que facilita a implementação e execução escalável de tarefas paralelas em sistemas computacionais distribuídos.

As infraestruturas utilizadas em nuvem foram de mini-clusters em CPUs e GPUs. A aplicação foi executada três vezes em cada mini-cluster e a mediana foi utilizada para resumir o resultado. A ferramenta de compilação do código utilizada foi o gpp versão 5.4.0 com a flag de otimização O3. Para o caso específico de GPUs foi utilizado o OpenCL versão 1.2. Por fim, para execução do PY-PITS foi utilizado Python versão 3.

Tabela 1. Grupos de máquinas

\begin{tabular}{|c|c|c|c|c|}
\hline Grupo & Máquinas & Núcleo de processamento & $\begin{array}{l}\text { Memória } \\
\text { RAM }\end{array}$ & $\begin{array}{l}\text { Custo } \\
\text { por hora }\end{array}$ \\
\hline CPU0 & $4 \times$ A9 & 64 vCPUs (Xeon E5-2670) & 448GB & $\mathrm{R} \$ 25,8960$ \\
\hline CPU1 & $1 \times$ D64 & 64 vCPUs (Xeon E5-2673 v3) & $256 \mathrm{~GB}$ & $\mathrm{R} \$ 10,1990$ \\
\hline CPU2 & $2 \times$ D64 & 128 vCPUs (Xeon E5-2673 v3) & $512 \mathrm{~GB}$ & $\mathrm{R} \$ 20,3980$ \\
\hline CPU3 & $4 \times$ D64 & 256 vCPUs (Xeon E5-2673 v3) & $1024 \mathrm{~GB}$ & $\mathrm{R} \$ 40,7960$ \\
\hline GPU0 & $2 \times$ NC6 & 2 Nvidia K80 & $112 \mathrm{~GB}$ & $\mathrm{R} \$ 05,0996$ \\
\hline GPU1 & $1 \times \mathrm{NC6S}$ & 1 Nvidia P100 & $112 \mathrm{~GB}$ & $\mathrm{R} \$ 06,8724$ \\
\hline GPU2 & $2 \times \mathrm{NC} 6 \mathrm{~S}$ & 2 Nvidia P100 & 224GB & $\mathrm{R} \$ 13,7448$ \\
\hline GPU3 & $1 \times \mathrm{NC} 24 \mathrm{~S}$ & 4 Nvidia P100 & $448 \mathrm{~GB}$ & $\mathrm{R} \$ 27,4896$ \\
\hline HET0 & $4 \times \mathrm{D} 16+2 \times \mathrm{NC} 6$ & $\begin{array}{c}\text { 64vCPUs (Xeon E5-2673 v3) } \\
2 \text { Nvidia K80 }\end{array}$ & 368GB & $\mathrm{R} \$ 15,2988$ \\
\hline HET1 & $1 \times \mathrm{NC6S}$ & $\begin{array}{c}6 \text { vCPUs (Xeon E5-2690) } \\
1 \text { Nvidia P100 }\end{array}$ & $112 \mathrm{~GB}$ & $\mathrm{R} \$ 06,8724$ \\
\hline HET2 & $1 \times \mathrm{NC6S}$ & $\begin{array}{c}4 \text { vCPUs (Xeon E5-2690) } \\
1 \text { Nvidia P100 }\end{array}$ & $112 \mathrm{~GB}$ & $\mathrm{R} \$ 06,8724$ \\
\hline
\end{tabular}

A Tabela 1 descreve os sistemas utilizados em nossos experimentos. O campo grupo descreve o dispositivo computacional utilizado para a execução do método. Aqui, CPU\# representa somente CPUs, GPU\# somente GPUs e HET\# representa ambos CPUs e GPUs. O campo máquinas descreve as máquinas virtuais utilizadas. O campo núcleo de processamento indica quantos núcleos virtuais (vCPUs) foram usados identificando o processador de cada vCPU e os aceleradores. Por fim, o campo custo por hora mostra qual o custo das máquinas. Este valor é informado pelo provedor de serviço da nuvem, e.g., no grupo HET2 foi usada uma máquina NC6S com 4 vCPUs e uma Nvidia P100 a um custo de $\mathrm{R} \$ 6,8724$ por hora de uso.

Os testes executados envolviam computar os parâmetros do método CMP para um dado de cerca de $950 \mathrm{MB}$, através do algoritmo heurístico evolutivo differential evolution (DE) [Storn and Price 1995] com cento e uma sementes e cento e uma gerações de evolução. Esses parâmetros combinados com outros específicos do processamento do 
dado, os quais a discussão está fora do escopo deste trabalho, garantiram uso de $100 \%$ das CPUs e das GPUs quase constante no decorrer da execução.

Por fim, duas métricas foram desenvolvidas para a avaliação dos resultados, sabendo que o maior tempo do programa é gasto computando interpolações. Essas métricas são: (i) interpolação por unidade monetária, que mede o potencial do algoritmo na máquina em relação ao custo monetário; e (ii) interpolações por unidade de tempo, que mede a velocidade que o programa computa interpolações.

\section{Resultados e discussões}

Na Tabela 2, pode-se comparar os resultados dos testes para cada grupo, contendo o tempo de execução em segundos, o custo total em Reais para a execução, a métrica de desempenho interpolações por segundo e a métrica financeira interpolações por Real. Por exemplo, o grupo GPU3 levou 158,24 segundos a um custo total de R \$1,21. Neste contexto, foram realizadas $1,34 \mathrm{E}+11$ interpolações por segundo e 1,75E+13 interpolações por Real.

Tabela 2. Resultados

\begin{tabular}{|c|c|c|c|c|}
\hline Grupo & Tempo & Custo & Interpolações/segundo & Interpolações/R $\$$ \\
\hline CPU0 & $3194,96 \mathrm{~s}$ & $\mathrm{R} \$ 22,98$ & $6,55 \mathrm{E}+09$ & $9,11 \mathrm{E}+11$ \\
\hline CPU1 & $4750,91 \mathrm{~s}$ & $\mathrm{R} \$ 13,46$ & $4,41 \mathrm{E}+09$ & $1,56 \mathrm{E}+12$ \\
\hline CPU2 & $2365,08 \mathrm{~s}$ & $\mathrm{R} \$ 13,40$ & $8,85 \mathrm{E}+09$ & $1,56 \mathrm{E}+12$ \\
\hline CPU3 & $1160,26 \mathrm{~s}$ & $\mathrm{R} \$ 13,15$ & $1,80 \mathrm{E}+10$ & $1,59 \mathrm{E}+12$ \\
\hline GPU0 & $0748,08 \mathrm{~s}$ & $\mathrm{R} \$ 01,06$ & $2,83 \mathrm{E}+10$ & $2,00 \mathrm{E}+13$ \\
\hline GPU1 & $0483,99 \mathrm{~s}$ & $\mathrm{R} \$ 00,92$ & $4,38 \mathrm{E}+10$ & $2,29 \mathrm{E}+13$ \\
\hline GPU2 & $0247,41 \mathrm{~s}$ & $\mathrm{R} \$ 00,94$ & $8,56 \mathrm{E}+10$ & $2,24 \mathrm{E}+13$ \\
\hline GPU3 & $0158,24 \mathrm{~s}$ & $\mathrm{R} \$ 01,21$ & $1,34 \mathrm{E}+11$ & $1,75 \mathrm{E}+13$ \\
\hline HET0 & $0737,71 \mathrm{~s}$ & $\mathrm{R} \$ 03,14$ & $2,87 \mathrm{E}+10$ & $6,57 \mathrm{E}+12$ \\
\hline HET1 & $0753,07 \mathrm{~s}$ & $\mathrm{R} \$ 01,44$ & $2,81 \mathrm{E}+10$ & $1,47 \mathrm{E}+13$ \\
\hline HET2 & $0490,62 \mathrm{~s}$ & $\mathrm{R} \$ 00,94$ & $4,32 \mathrm{E}+10$ & $2,26 \mathrm{E}+13$ \\
\hline
\end{tabular}

Os resultados mostram que apesar das máquinas A9 utilizadas no grupo CPU0 serem indicadas pelo provedor para a execução de código de alto desempenho, para a aplicação executada, o custo benefício das máquinas D64 - dos grupos CPU1, CPU2 e CPU3 - mostrou-se superior. Ao explorar a escalabilidade do código, permitiu-se obter resultados mais rápidos e mais baratos aumentando o número de instâncias D64.

Ao usar máquinas CPU com máquinas GPU - grupo HET0 - existe uma perda considerável na métrica interpolações por real, pois as CPUs aumentaram o preço em uma escala maior que aumentam o desempenho. No grupo HET1, não vale a pena o uso de todos os núcleos da CPU para tentar ajudar a GPU, pois a GPU fica subutilizada pela demora maior ao receber dados, enquanto ao usar apenas 4 núcleos - no grupo HET2 -, não se percebe diferença para o uso somente da GPU.

As GPUs apresentam os melhores resultados tanto em tempo quanto em custo benefício, vide que o grupo GPU1 mostra o melhor resultado em Interpolações/R $\$$. Comparando os grupos GPU1, GPU2 e GPU3, o aumento no número de GPUs mostra uma melhora no tempo, porém existe também uma perda de custo benefício. Ape- 
sar desta aplicação não escalar com o preço, o aumento de desempenho ainda é considerável. Esta escalabilidade de CPUs e GPUs se dá devido à característica de algoritmo embaraçosamente paralelo.

Para adquirirmos a máquina NC24S, seria necessário o investimento de cerca de 315 mil Reais, baseando-se nos preços da Dell. Supondo um projeto de 48 meses, vamos agora verificar quanto teria que ser o uso da máquina para compensar o investimento em hardware, desconsiderando o investimento em TI para a manutenção e também o custo de Internet, além disso supõe-se que, usando a nuvem, o valor inicial da máquina está em um investimento fixo de $0,5 \%$ ao mês, enquanto ao adquirir o hardware, ele será vendido no final do projeto com uma depreciação entre $20 \%$ a $36 \%$ ao ano.

Dado um consumo elétrico de $3500 \mathrm{~W}$ para a máquina e o sistema de refrigeração com um preço de energia elétrica de $\mathrm{R} \$ 0,6 / \mathrm{kWh}$ contra o gasto de $\mathrm{R} \$ 27,4896$ por hora da máquina NC24S em nuvem, conclui-se que seria necessário usar a máquina $70 \%$ do tempo para compensar o investimento em hardware, caso contrário, a nuvem é mais economicamente viável.

\section{Conclusão}

Neste trabalho investiga-se o uso da nuvem computacional Azure, da Microsoft, para a execução de algoritmos no processamento sísmico. Sendo que nessa nuvem o desempenho do algoritmo do método CMP é analisado para cada tipo de hardware utilizado. Os resultados mostram uma redução do custo/benefício ao utilizar máquinas do grupo CPU ou do grupo HET contra o uso de máquinas GPU, concluindo assim que máquinas do grupo GPU são de fato as melhores escolhas para o tipo de aplicação. Além disso, observa-se que em aumentar o número de núcleos de CPU ou GPUs o desempenho melhore proporcionalmente, ou seja, o tempo de execução diminui ao passo que o aumenta o número de núcleos de processamento, dessa forma, mostra-se que existe escalabilidade no programa. Por fim, caso a nuvem seja usada menos que $70 \%$ no período o seu uso é mais vantajoso que a compra de novas máquinas físicas.

\section{Agradecimentos}

Os autores agradecem à FAPESP/CEPID \#2013/08293-7 e à Petrobras pela ajuda financeira, ao laboratório High Perfomance Geophysics (HPG) pelo suporte computacional e à Microsoft pelo acesso à Azure.

\section{Referências}

Borin, E., Benedicto, C., Rodrigues, I. L., Pisani, F., Tygel, M., and Breternitz, M. (2016). PY-PITS: A scalable python runtime system for the computation of partially idempotent tasks. In 2016 International Symposium on Computer Architecture and High Performance Computing Workshops (SBAC-PADW). IEEE.

Storn, R. and Price, K. (1995). Differential evolution-a simple and efficient adaptive scheme for global optimization over continuous spaces. international computer science institute, berkeley. Technical report, CA, 1995, Tech. Rep. TR-95-012.

Yilmaz, O. (2001). Seismic Data Analysis, volume 10 of Investigations in Geophysics. Society Of Exploration Geophysicists, Tulsa, USA, 2 ed edition. 